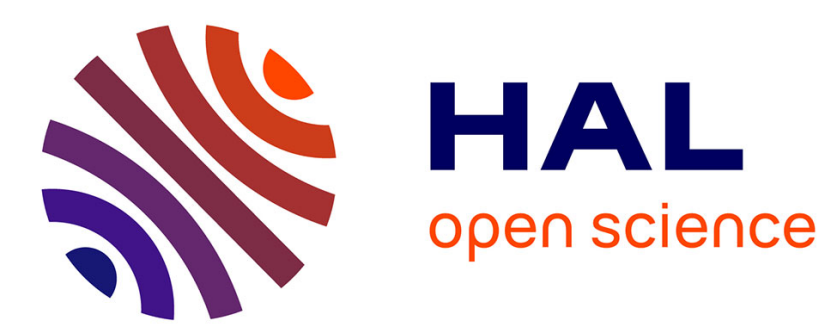

\title{
Wood elastic characterization from a single sample by resonant ultrasound spectroscopy
}

Roberto Longo, Thomas Delaunay, Didier Laux, Mohammed El Mouridi, Olivier Arnould, Emmanuel Le Clezio

\section{- To cite this version:}

Roberto Longo, Thomas Delaunay, Didier Laux, Mohammed El Mouridi, Olivier Arnould, et al.. Wood elastic characterization from a single sample by resonant ultrasound spectroscopy. Ultrasonics, 2012, 52, pp.971-974. 10.1016/j.ultras.2012.08.006 . hal-00742901

\section{HAL Id: hal-00742901 https://hal.science/hal-00742901}

Submitted on 17 Oct 2012

HAL is a multi-disciplinary open access archive for the deposit and dissemination of scientific research documents, whether they are published or not. The documents may come from teaching and research institutions in France or abroad, or from public or private research centers.
L'archive ouverte pluridisciplinaire HAL, est destinée au dépôt et à la diffusion de documents scientifiques de niveau recherche, publiés ou non, émanant des établissements d'enseignement et de recherche français ou étrangers, des laboratoires publics ou privés. 


\title{
Wood elastic characterization from a single sample by resonant ultrasound spectroscopy
}

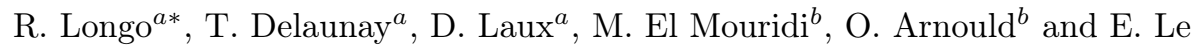 \\ Clézio $^{a}$ \\ ${ }^{a}$ Institut d'Electronique du Sud (IES), Université Montpellier 2, UMR CNRS 5214, CC084 \\ Place Eugène Bataillon, 34095 Montpellier, France \\ ${ }^{b}$ Laboratoire de Mécanique et Génie Civil (LMGC), Université Montpellier 2, UMR CNRS \\ 5508, CC048 Place Eugène Bataillon, 34095 Montpellier, France
}

\begin{abstract}
The goal of this paper is to propose an experimental method allowing the identification of the complete elastic tensor of anisotropic biological materials such as wood using only one sample. To do so, two complementary methods are used. First, the wood eigen-directions are defined from a sample of spherical shape that is then cut into a cube in a way to perform resonant ultrasound spectroscopy (RUS). The method is successfully applied on a reference beech sample with known orthotropic directions. A comparison of the identified elastic constants with those from the literature and some inferred from ultrasonic transmission measurements is given.
\end{abstract}

Keywords: Resonant ultrasound spectroscopy, spherical and cubic samples, wood, elastic constants

\section{Introduction}

Wood is a natural visco-elastic composite material usually considered to behave like an orthotropic medium at the millimeter scale with eigen-directions, or natural symmetry axes, that are theoretically parallel to the visible anatomical ones of the tree: radial $\mathbf{R}$ (1), tangential $\mathbf{T}$ (2) and longitudinal $\mathbf{L}$ (3) in cylindrical coordinates, see Fig. 1. This material is difficult to characterize due to its biological origin yielding anisotropic behavior, strong variability (especially within a tree), heterogeneity and sensitivity to moisture content, temperature and loading time. Difficulties then occur in determining all the components of the elastic tensor $C_{i j}$ (in Voigt notation where $C_{44}=C_{2323}, C_{55}=C_{1313}$ and $C_{66}=C_{1212}$ ) of wood materials, especially the off-diagonal terms that depend on the Poisson's ratios. This leads to underuse and/or misuse this material,

*E-mail address: roberto.longo@univ-montp2.fr 


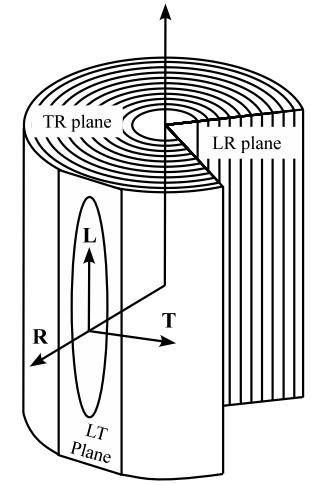

Figure 1: Symmetry axes of a tree trunk.

hence predicting accurately its behavior for given environmental conditions is still an open question.

Classical characterization methods for anisotropic materials usually need the machining of several samples along different directions. However, due to the strong variability within a single tree, the consistence of the corresponding constants is not ensured. The reduction of the number of samples used for the determination of all the elastic constants is then a crucial point for wood. Quasi-static measurements on a single cubic sample of wood have been done $[1,2]$. However, the development of fast measurement techniques is important to increase the number of available data for a relevant statistical analysis. Fast measurements can be obtained by using different techniques based on ultrasonic (or acoustic) wave propagations in direct contact $[3,4]$ or in a coupling medium (e.g., water) with a protective coating if dry wood properties have to be measured $[5,6]$. These techniques allow the determination of the diagonal terms of the elastic tensor on a unique parallelepipedic sample [7] but, except in some cases with a polyhedral [8] or spherical samples $[6,9]$, they usually require other samples, and/or coupling with other kind of measurements, for the determination of off-diagonal terms [10, 11, 12, 13, 14].

Resonant ultrasound spectroscopy (RUS) could offer a good alternative because the determination of the entire specimen elastic constants can be obtained from only one sample thanks to non contact measurements of its resonant frequencies. It has already been applied to isotropic [15] or anisotropic materials presenting a known symmetry [16] but its application becomes more complex when testing materials of unknown symmetry and with high attenuation. It was first used on wood by Schubert [17] but only for the determination of one shear modulus by using the two lowest resonance frequencies.

In this paper we propose an experimental study combining methods allowing the determination of the wood symmetry and that of its elastic constants. The three symmetry directions of the material are first identified from a spherical 
specimen by ultrasonic tomography [9]. The sample is then cut into a cubic shape and subjected to RUS measurements. The method is validated on a beech specimen (Fagus Sylvatica L.) whose full elastic tensor is eventually determined and compared to literature values and to some obtained by ultrasonic velocimetry.

\section{Method}

\subsection{Preparation of the sample}

To apply the RUS method to cubic samples one has to know the material symmetry and its eigen-directions should preferably be perpendicular to the cube faces. However, wood characteristics undergo modifications form the center of the tree to its periphery. In particular, the radius of the annual ring is gradually increasing. To overcome this problem, we propose here to couple RUS measurement with a preliminary determination of the eigen/orthotropic directions of wood specimen by using ultrasonic tomography in a sphere sample [9], see Fig. 2. This technique is similar to the one used by Bucur and Rasolofosaon [6] except that direct contact measurements are perfomed in the present case. Once the main orientations $\mathbf{R}, \mathbf{T}$ and $\mathbf{L}$ are found, a cubic sample with the surfaces in agreement with the orthotropic symmetry axes can directly be cut from the sphere. A previous study has shown that the estimated error on the alignment of the symmetry axes to the normal of the cube surfaces is around $3^{\circ}[9]$. Simulations proved that this leads to variations less than $3 \%$ on the resonant frequencies of the cube presented here.

\subsection{Preliminary tests: ultrasonic velocimetry}

Once the cubic sample is available, pulse measurements in transmission are performed in the $\mathbf{R}, \mathbf{T}$ and $\mathbf{L}$ directions using contact transducers: Panametrics V103 (center frequency: $1 \mathrm{MHz}$, diameter: $13 \mathrm{~mm}$ ) and Panametrics V151 (center frequency: $0.5 \mathrm{MHz}$, diameter: $26 \mathrm{~mm}$ ) for longitudinal and transverse measurements respectively. To improve the signal to noise ratio, $50 \mu \mathrm{s}$ transmitted signals captured by the receiving transducer are acquired 100 times by means of a digital oscilloscope (LeCroy WaveJet 334) and averaged. The signal at the receiver without the sample (transmission with the surfaces of the transducers in contact) is also acquired and the wave time of travel through the sample in the directions $\mathbf{R}, \mathbf{T}$ and $\mathbf{L}$ is estimated implementing a cross correlation between these two signals. The sound velocities are derived thanks to the measurement of the thickness of the samples. Knowing the material density, it is then possible to estimate the diagonal values $C_{i i, i=1 \ldots 6}$ of the elastic tensor [18] (Table 1).

\subsection{RUS measurements}

As mentioned above, RUS is a technique based on the measurement of the different resonant frequencies of the specimen under test. A typical set-up includes an ultrasonic excitation sent out by a piezoelectric (PZT) transducer put 
in contact with the sample. The resonant frequencies describing the mechanical vibrations of the sample under test can be collected with another piezoelectric probe [19] or by means of optical techniques (e.g., Laser Doppler Vibrometry, holography) [16, 20, 21].

In this work, an arbitrary wave generator (Agilent 33220A) sends out an electric signal to a PZT transducer placed at the corner of the sample. Care was taken to choose the correct probe, in order to avoid the convolution of the transducer resonant frequencies with the ones of the wood sample. In our case, the transducer employed during the RUS measurements had its first resonance at $200 \mathrm{kHz}$. The electric signal was a $10 \mathrm{~V}$ peak-to-peak chirp of $20 \mathrm{~ms}$

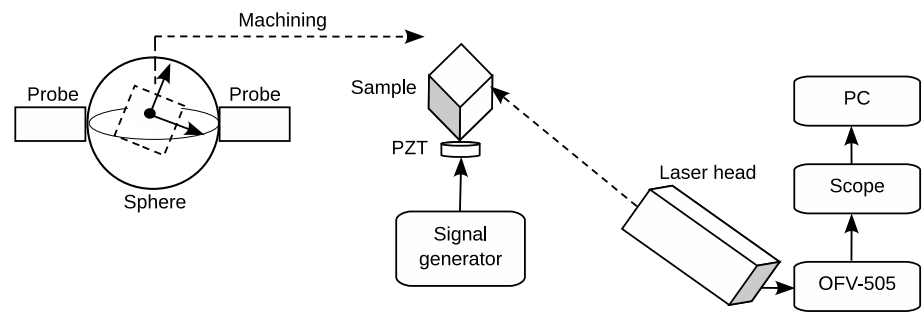

Figure 2: Whole experimental set-up from the identification of the material eigen directions on a sphere to the RUS measurements. The cubic sample cut from the sphere is excited by a PZT sending out a chirp signal. The induced vibrations are measured by the Laser Doppler Vibrometer.

with a bandwidth ranging from $20 \mathrm{kHz}$ to $80 \mathrm{kHz}$. This choice of the shape of the excitation signal significantly reduces the measurement time. The induced mechanical vibrations in the structure and thus the associated resonant frequencies were measured by means of a Laser Doppler Vibrometer (Polytec OFV-505) with laser light aiming at one corner of the RT face of the sample under test where most of the eigen modes possess a significant displacement (see Fig. 2). To improve the experimental signal to noise ratio, an auto-correlation analysis has been performed [22] leading to a filtered power spectrum depicted in Fig. 3 and obtained for the beech sample.

Knowing the resonant frequencies of the samples gives the possibility to estimate the elastic tensor $C_{i j}$ by solving the inverse problem. This task was supported by simulations able to calculate the theoretical values of these frequencies, with an initial tensor $C_{i j}$ given in input (direct problem). In the next paragraph some insights of these techniques are briefly reported.

\subsection{Solving the direct and inverse problem}

In order to evaluate the theoretical resonant frequencies of the samples under test, the direct problem $[23,16]$ is solved with initial values of the elastic tensor $C_{i j}^{0}$ computed using the empirical relationships developped by Guitard and Amri [1] knowing the wood density. They represent a good starting point for our investigation as they offer an order of magnitude concerning the elastic coefficients of the wood sample under test. 


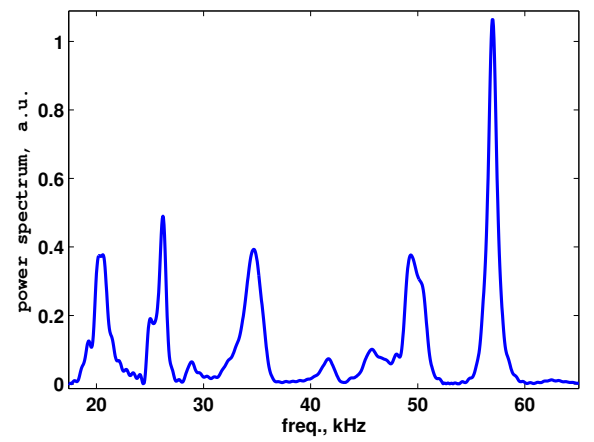

Figure 3: Measured resonant frequencies for the beech sample.

Hence, the inverse problem is solved by modifying the initial values of the $C_{i j}$ tensor to fit the measured resonant frequencies obtained in Section 2.3. This algorithm enables the determination of the elastic constants by minimizing the distance $\Delta_{m c}$ between the measured $(m)$ resonance frequencies and the computed $(c)$ ones using the Simplex routine [16]:

$$
\Delta_{m c}=\frac{\sum_{i}\left|f_{\text {measured }}^{i}-f_{\text {computed }}^{i}\right|}{f_{\text {measured }}^{i}} .
$$

Due to the orthotropic symmetry of the sample, nine constants of the $C_{i j}$ tensor had to be identified for the cubic sample under test [18]: Longitudinal constants $\left(C_{11}, C_{22}\right.$ and $\left.C_{33}\right)$, off-diagonal constants $\left(C_{12}, C_{13}\right.$ and $\left.C_{23}\right)$ and shear constants $\left(C_{44}, C_{55}\right.$ and $\left.C_{66}\right)$.

With such number of parameters, the convergence to the correct solution could be difficult to obtain. For this reason a characterization protocol is proposed. To do so, a sensitivity study has been performed in a way to select resonances sensitive to only one elastic constant. As an example, the first resonance has been proved to be highly sensitive to $C_{66}$. In a first phase of the convergence procedure, the corresponding parameters are separately identified while fixing the other ones. In a second phase, the constants with influence on multiple resonances are deduced from a global fit. The results of this procedure are given in the next section.

\section{Results and discussion}

To validate the proposed experimental characterization method, the whole protocol is here applied to a beech specimen. Indeed, beech wood has the advantages to be a quite homogeneous, orthotropic and already well characterized, allowing the comparison of our results with those from the literature [1, 3, 12]. The determination of the material symmetry thanks to the tomography of a sphere led to a $20.91 \times 21.06 \times 21.11 \mathrm{~mm}^{3}$ cubic shaped specimen. The sample 
thicknesses were measured using a micrometer with a precision of $10 \mu \mathrm{m}$. Density was then assessed as mass divided by volume yielding a value of $717 \mathrm{~kg} \cdot \mathrm{m}^{-3}$ with a precision of $1 \%$. All measurements have been done in room conditions (i.e., around $20^{\circ} \mathrm{C}$ and $30 \% \mathrm{RH}$ ) and the results of the velocity measurements of Section 2.2 have been used to estimate the diagonal constants of the elastic tensor (Table 1) from the classical relationship $C=\rho V^{2}$.

To identify the elastic tensor thanks to RUS measurements, the iterative computation previously described is then performed on the first nine resonances of the sample ranging between $20 \mathrm{kHz}$ and $70 \mathrm{kHz}$, see Fig. 3. The experimental and final numerical resonance frequencies are given in Table 2.

Table 1: Estimated elastic tensor components (in GPa) for the beech wood sample, with the initial values $C_{i j}^{0}$ used for RUS identification between brackets, and comparison to litterature data on beech (m.c. = moisture content). The standard deviations for velocimetry measurements are given in parenthesis.

\begin{tabular}{|c|cc|ccc|}
\hline Parameters & Trans & RUS $\left[C_{i j}^{0}\right]$ & $\begin{array}{c}\text { Hering et al. } \\
{[12]}\end{array}$ & $\begin{array}{c}\text { Bucur } \\
{[3]}\end{array}$ & $\begin{array}{c}\text { L.E.M.T.A. } \\
{[1]}\end{array}$ \\
\hline$\rho\left(\mathrm{kg} / \mathrm{m}^{3}\right)$ & \multicolumn{2}{|c|}{$717(7)$} & $691-711$ & 674 & 676 \\
m.c. $(\%)$ & \multicolumn{2}{|c|}{$\approx 7.5$} & 12.3 & - & 12 \\
\hline$C_{11}$ & $3.6(0.1)$ & $3.5[2.9]$ & 2.5 & 3.4 & 3.4 \\
$C_{22}$ & $1.7(0.1)$ & $1.8[1.7]$ & 0.8 & 1.4 & 1.4 \\
$C_{33}$ & $20.3(0.5)$ & $19.5[17.2]$ & 16.0 & 17.9 & 18.7 \\
$C_{12}$ & - & $1.3[1.2]$ & 0.7 & 1.0 & 1.6 \\
$C_{13}$ & - & $1.8[1.7]$ & 1.7 & 5.2 & 3.4 \\
$C_{23}$ & - & $1.6[1.3]$ & 1.2 & 2.6 & 2.1 \\
$C_{44}$ & $0.95(0.1)$ & $1.0[1.1]$ & 0.8 & 1.0 & 0.9 \\
$C_{55}$ & $1.5(0.2)$ & $1.4[1.3]$ & 1.3 & 1.4 & 1.5 \\
$C_{66}$ & $0.55(0.1)$ & $0.5[0.4]$ & 0.5 & 0.3 & 0.4 \\
\hline
\end{tabular}

In order to estimate the efficiency of the fitting procedure, the deviation $\Delta_{m c}$ between the measured resonant frequencies and the computed ones is discussed. First of all, as shown in Table 2, the maximum deviation is $1.5 \%$ and the mean value on the nine resonances is $0.67 \%$, indicating a very good quality of the convergence. Then, the individual deviations may be linked to the precision that one may obtain during the identification of the different constants. As previously mentioned, the first resonance is highly sensitive to $C_{66}$ and the very good agreement between the corresponding measured and computed frequencies yields a good confidence in the estimated elastic constant value. The whole set of elastic constants estimated for the beech cubic sample under test is presented in Table 1 together with other authors' measurements. It shows that the diagonal values $C_{i i, i=1 \ldots 6}$ of this elastic tensor agree with the ones obtained from ultrasonic transmission measurements. Indeed, the relative deviations $\delta_{i i}$ are: $\delta_{11}=-2.86 \%, \delta_{22}=+5.55 \%, \delta_{33}=-4.1 \%, \delta_{44}=+5.0 \%, \delta_{55}=-7.14 \%$ and $\delta_{66}=-10.0 \%$. The highest deviations are obtained for the shear moduli where 
Table 2: Measured and predicted resonance frequencies for the beech wood cubic sample.

\begin{tabular}{|c|c|c|c|}
\hline Mode & $\begin{array}{c}\text { Measured frequencies } \\
(\mathrm{Hz})\end{array}$ & $\begin{array}{c}\text { Computed frequencies } \\
(\mathrm{Hz})\end{array}$ & $\begin{array}{c}\Delta_{m c} \\
(\%)\end{array}$ \\
\hline 1 & 20150 & 20114 & 0.2 \\
2 & 26200 & 26210 & $<0.1$ \\
3 & 28850 & 29263 & 1.4 \\
4 & 34700 & 34317 & 1.1 \\
5 & 41700 & 41677 & $<0.1$ \\
6 & 45850 & 45466 & 0.8 \\
7 & 49250 & 48975 & 1.5 \\
8 & 57000 & 57409 & 0.7 \\
9 & 62500 & 62518 & $<0.1$ \\
\hline
\end{tabular}

ultrasonic transmission measurements are less accurate. This already offers an important validation of the RUS technique application to wood samples. The comparison with the initial values $C_{i j}^{0}$ used for the indentification procedure shows that most of the final RUS values are in good agreement with deviations less than $10 \%$. However, some parameters underwent significant modifications and a maximum deviation of $20 \%$ is registered for the $\delta_{66}, \delta_{11}$ and $\delta_{23}$ being of the same order of magnitude. This can be explained by the fact that the estimated constants $C_{i j}^{0}$ given by the empircial relationships developped by Guitard and Amri [1] are mean characteristics obtained from various samples and only take into account density effects on elastic properties.

Table 1 also presents full sets of elastic constants found in the literature for beech wood samples $[1,18,12]$. Being consistent, they however present some discrepancies. As previously noticed, wood materials are known to be inhomogeneous and to undergo property modifications inside a single species and even inside a single tree leading to final tensor inconsistencies. Moreover, the moisture content of the sample also influences the material stiffness. As a consequence, the relatively good agreement between the beech properties presented in this paper and those from the literature allows us to have a good confidence in the further application of the RUS method to the characterization of wood.

\section{Conclusion}

In this paper, an experimental characterization protocol of wood stiffness constants is presented. It couples two ultrasonic methods allowing the determination of the material symmetry by ultrasonic tomography of a sphere and the identification of the orthotropic tensor thanks to Resonant Ultrasound Spectroscopy. In a way to validate its application to complex materials such as wood, the characterization of a beech reference sample has been performed. The material properties were compared with success to values from the literature and to some obtained by ultrasonic velocimetry. Beech being of low symmetry, these 
good results prove that the RUS method is a promising tool to characterize wood species in different environmental conditions ranging from dry to green wood. Future research could evaluate the possibility to avoid the use of a sphere in order to apply the RUS directly on cubic samples cut in the anatomical directions. This task could also involve the use of Scanning Laser Vibrometer (SLDV) and/or holographic techniques to experimentally determine the sample modes shapes.

\section{Acknowledgements}

This project was supported by the Labex NUMEV (Universite Montpellier 2, France).

\section{References}

[1] D. Guitard, F. El Amri, Modèles prévisionnels de comportement élastique tridimensionnel pour les bois feuillus et les bois résineux, Annales des Sciences Forestières 44 (1987) 335-358.

[2] B. Hassel, P. Berard, C. Moden, L. Berglund, The single cube apparatus for shear testing - Full-field strain data and finite element analysis of wood in transverse shear, Composites Science and Technology 69 (2009) 877-882.

[3] V. Bucur, R. R. Archer, Elastic constants for wood by an ultrasonic method, Wood Science and Technology 18 (1984) 255-265.

[4] C. Kohlhauser, C. Hellmich, C. Vitale-Brovarone, A. R. Boccaccini, A. Rota, J. Eberhardsteiner, Ultrasonic characterisation of porous biomaterials across different frequencies, Strain 45 (2009) 34-44.

[5] C. Preziosa, M. Mudry, J. Launay, F. Gilletta, Determination of the elasticity coefficients of wood with a goniometric acoustical method, C.R.A.S. Serie II 293 (1981) 91-94.

[6] V. Bucur, P. Rasolofosaon, Dynamic elastic anisotropy and nonlinearity in wood and rock, Ultrasonics 36 (1998) 813-824.

[7] D. Keunecke, W. Sonderegger, K. Pereteanu, T. Luethi, P. Niemz, Determination of Young's and shear moduli of common yew and Norway spruce by means of ultrasonic waves, Wood science and technology 41 (2007) 309-327.

[8] M. Francois, G. Geymonat, Y. Berthaud, Determination of the symmetries of an experimentally determined stiffness tensor, application to acoustic measurements, International Journal of Solids and Structures 35 (1998) 4091-4106. 
[9] M. El Mouridi, T. Laurent, L. Brancheriau, O. Arnould, A. Famiri, A. Hakam, J. Gril, Searching for material symmetries in the burr wood of thuja by a direct contact ultrasonic method on spherical samples, Maderasciencia y tecnologia 13 (2011) 285-296.

[10] S. Dahmen, H. Ketata, M. Ben Ghozlen, B. Hosten, Elastic constants measurement of anisotropic Olivier wood plates using air-coupled transducers generated Lamb wave and ultrasonic bulk wave, Ultrasonics 50 (2010) 502-507.

[11] J. Fortineau, E. Le Clezio, M. Vander, J. Vallete, Ultrasonic characterisation of wood, in: 1st Mediterranean congress on acoustics, Salé, Morocco, 2010 .

[12] S. Hering, D. Keunecke, P. Niemz, Moisture-dependent orthotropic elasticity of beech wood, Wood Science and Technology (2011).

[13] R. Goncalves, A. Trinca, D. Pellegrino Cerri, Comparison of elastic constants of wood determined by ultrasonic wave propagation and static compression test, Wood and Fider Science 43 (2011) 64-75.

[14] C. Kohlhauser, C. Hellmich, Determination of Poisson's ratios in isotropic, transversely isotropic, and orthotropic materials by means of combined ultrasonic-mechanical testing of normal stiffnesses: Application to metals and wood, European Journal of Mechanics A-Solids 33 (2012) 82-98.

[15] M. Visscher, A. Migliori, T. Bell, R. Reinert, On the normal modes of free vibration of homogeneous and anisotropic elastic objects, Journal of the Acoustical Society of America 91 (1990) 2154-2161.

[16] T. Delaunay, E. Le Clezio, M. Guennou, H. Dammak, M. Thi, G. Feuillard, Full tensorial characterization of $\mathrm{PZN}-12 \% \mathrm{PT}$ single crystal by resonant ultrasound spectroscopy, IEEE Transactions on Ultrasonics Ferroelectrics and Frequency Control 55 (2008) 476-488.

[17] S. Schubert, D. Gsell, J. Dual, M. Motavalli, P. Niemz, Rolling shear modulus and damping factor of spruce and decayed spruce estimated by modal analysis, Holzforschung 60 (2006) 78-84.

[18] V. Bucur, Acoustics of Wood, Springer-Verlag, 2nd edition, 2006.

[19] J. Maynard, Resonant ultrasound spectroscopy, Physics Today 49 (1996) $26-31$.

[20] L. Drain, The laser doppler technique, John Wiley, 1980.

[21] F. Joud, F. Laloe, M. Atlan, J. Hare, M. Gross, Imaging a vibrating object by Sideband Digital Holography, Optics express 17 (2009) 2774-2779.

[22] J. Bendat, A. Piersol, Random data: analysis and measurement procedures, Wiley-Interscience, 1971. 
[23] I. Ohno, Rectangular parallellepiped resonance method for piezoelectriccrystals and elastic-constants of alpha-quartz, Physics and chemistry of minerals 17 (1990) 371-378. 